of ACPA. This initial development of auto-immunity appears to be independent of the disease-predisposing HLA-molecules. In most patients, this early event generates a polyclonal yet limited, mostly low-level autoantibody response that can be present for many years in the absence of clinical symptoms. Upon a putative second trigger, the ACPA epitope recognition repertoire broadens, more isotypes are being used, and ACPA serum levels rise. This is followed by precipitation of disease and is likely associated with the presence of the predisposing HLA-molecules. While the nature of this second trigger is presently unknown, the second event that initiates the broadening of the auto-immune response, in particular the citrulline-specific immune response, could mark a crucial moment upon which the auto-immune response becomes self-perpetuating and, potentially, irreversible.

Despite the many facets of ACPA revealed in the past two decades summarized above, it is not known how a breach of tolerance towards citrullinated proteins is mediated, or how ACPA-producing B-cells emerge.

Provision of T-cell help is crucial to convey the ability to B cells to modify the B cell receptor through somatic hypermutation. At present, it is unknown how ACPAor other Anti-Modified Protein Antibody (AMPA)-producing B cells are "helped" by CD4+ Thelper cells, but it is often speculated that an auto-reactive T-cell response is crucial for their appearance. Our recent data show that such help could be provided by T-cells recognizing foreign proteins that have undergone a post-translational modification. In mice, AMPA-responses recognizing modified self-proteins are readily induced by immunization with modified proteins of nonselforigin. This is explained by the observation that the murine AMPA-response was, both at the monoclonal- and polyclonal level, highly cross-reactive towards multiple modified proteins, including proteins of self- and foreign origin. A similar observation was made analyzing the AMPA response in sera from RA patients. These data are important as the cross-reactive nature of AMPA could explain how autoreactive B-cell responses against PTM self-proteins can be induced by exposure to PTM foreign proteins thereby providing new insights on the breach of autoreactive B-cell tolerance.

Taken together, the analysis of the fine-specificity and recognition pattern of antibodies against modified proteins in RA during different phases of disease, together with detailed studies on the the identification, isolation and phenotypic characterization of auto-reactive B cells that express AMPA starts to shed light on the earliest phases of autoimmunity in RA.

Disclosure of Interest: None declared

DOI: 10.1136/annrheumdis-2017-eular.7218

\section{SP0146 CAN WE PREDICT WHO IS GOING TO DEVELOP RHEUMATOID ARTHRITIS?}

D. Van Der Woude. Rheumatology, Leiden University Medical Center, Leiden, Netherlands

To accurately predict disease development can be considered the "holy grail" of risk factor research. It holds the potential to employ preventive treatment thereby nipping RA in the bud.

This presentation will review familial risk in RA and the underlying genetic risk factors, as well as environmental risk factors for disease. Autoantibodies are a potent prognostic marker when it comes to the risk of developing RA, and play a key role in current pathophysiological hypotheses. The newest players in the autoantibody field, and latest concepts of how the various risk factors contribute to disease onset will be discussed.

Disclosure of Interest: None declared

DOI: 10.1136/annrheumdis-2017-eular.7141

\section{SP0147 CAN WE PREVENT THE ONSET OF RHEUMATOID ARTHRITIS IN HIGH RISK INDIVIDUALS?}

K.D. Deane. Division of Rheumatology, University of Colorado Denver, Aurora, United States

Multiple studies have demonstrated that rheumatoid arthritis (RA) related biomarkers can identify individuals without inflammatory arthritis who are at high-risk for the future development of clinically apparent synovitis and classified RA. These findings have led to the development of several prevention trials in RA that have either been completed, or are underway. With these exciting developments as background, this lecture will discuss multiple aspects of RA prevention including the role of biomarkers and other factors in developing robust prediction models for future RA, and methods to identify individuals before they develop RA. In addition, this lecture will discuss specific preventive approaches to RA such as clinical trial design and choice of preventive interventions that are based on our growing understanding of the mechanisms of RA development.

Disclosure of Interest: None declared

DOI: 10.1136/annrheumdis-2017-eular.7235

\section{SP0148 PATHOPHYSIOLOGY OF ESTABLISHED RA SYNOVITIS}

B. Lauwerys. Department of Rheumatology, Université catolique de Louvain, Brussels, Belgium

Access to synovial tissue through - minimally invasive - synovial biopsy procedures led to the implementation of new translational approaches to our understanding of established RA. In this lecture, we will illustrate how the identification of different synovial pathotypes and related molecular pathways translated into clinically relevant phenotypes, such as disease severity or response to therapy. Validation of these concepts in ongoing large-scale multi-centric trials will be key to the integration of synovial assessment tools in clinical practice.

Disclosure of Interest: B. Lauwerys Shareholder of: DNALytics DOI: 10.1136/annrheumdis-2017-eular.7282

\section{FRIDAY, 16 JUNE 2017 Laboratory course - from the clinic to the lab and back II}

\section{SP0149 NEW TRENDS IN BIOMARKERS IN INFLAMMATORY JOINT DISEASES}

E. Feist. Rheumatology, Charite University Hospital, Berlin, Germany

This lecture provides an overview on new developments in biomarker research and standardization in inflammatory joint diseases. The presentation includes an introduction of established and new biomarkers in serum and synovial fluid as well as methods for their detection. Furthermore, an overview on different modifications of auto-antigens (including citrullinated and carbamylated isoforms) and their role in immune response and pathogenesis of disease will be given. The diagnostic performance of new and established biomarkers will be discussed including antibodies against modified antigens also illustrated by difficult to diagnose cases. In this context, special attention will be attributed to the predictive value of biomarkers for diagnosis of disease and treatment response.

Disclosure of Interest: None declared

DOI: 10.1136/annrheumdis-2017-eular.7200

\section{FRIDAY, 16 JUNE 2017}

\section{Switching T on and off: how $T$ cells drive and regulate chronic inflammation}

\section{SP0150 TH17 CELLS DRIVE AND REGULATE TISSUE INFLAMMATION}

V. Kuchroo on behalf of Basic and Translation Science. Evergrande Center for Immunologic Diseases, Harvard Medical School, Boston, United States

Recently a subset of interleukin (IL)-17-producing $T$ cells $\left(T_{H} 17\right)$ distinct from $T_{H} 1$ or $T_{H} 2$ cells was described and shown to have a crucial role in the induction of autoimmune tissue injury. Accumulating data suggests that there are three distinct steps in Th17 differentiation: Induction, Amplification and Stabilization mediated by distinct cytokines. Whereas TGF-b + IL-6 or IL- $1+$ IL- 6 induces them, IL-21 amplifies Th17 cells, IL-23 stabilizes the phenotype of Th17 cells. Loss of any of the cytokines (TGF- $\beta$, IL-1, IL-6, IL-21 or IL-23) in the pathway results in a defect in generation of Th17. However not all Th17 cells are pathogenic and induce autoimmunity, IL-23 is a key cytokine that induces pathogenicity in Th17 cells (Lee et al., 2012). Using expression profiling at very high temporal resolution, novel computational algorithms and innovative nanowire based "knock-down" approaches, we have developed a regulatory network that governs the development of Th17 cells. In addition to high-density temporal microarray analysis, we have performed single-cell RNA-seq of Th17 cells in order to characterize cellular heterogeneity, identify subpopulations, functional states and learn how gene expression variation affects Th17 effector functions. We have identified novel regulators of Th17 cells both in vivo and in vitro that do not affect Th17 differentiation but affect pathogenic vs. non-pathogenic functional states of Th17 cells. Some of the regulators that make Th17 cells non-pathogenic are also utilized by CD8+ T cells to induce T cell "exhaustion" or "dysfunction". These novel inhibitory molecules cooperate with other known "check-point" co-inhibitory receptors to suppress anti-tumor immunity.

Disclosure of Interest: None declared

DOI: 10.1136/annrheumdis-2017-eular.7254

\section{SP0151 SWITCHING OFF UNWANTED IMMUNE RESPONSES: THE MECHANISM OF ANTIGEN-SPECIFIC IMMUNOTHERAPY WITH T CELL EPITOPES}

D.C. Wraith. Institute of Immunology \& Immunotherapy, University of Birmingham, Birmingham, United Kingdom

Control of autoimmune and allergic conditions can be reinforced by tolerance induction with peptide epitopes; this presentation will focus on the mechanisms involved. Peptides must mimic naturally processed epitopes. Peptide induced peripheral tolerance is characterised by the generation of anergic, IL-10 secreting $\mathrm{CD}^{+}{ }^{+}$T-cells with regulatory function. CD4 ${ }^{+}$T-cells become anergic following their first encounter with peptide. The loss of proliferative capacity correlates with a cytokine switch from a pro-inflammatory to a phenotype characterised by secretion 